\title{
Robust optimal design of a nonlinear dynamic vibration absorber combining sensitivity analysis
}

\author{
R.A. Borges ${ }^{\mathrm{a}}$, A.M.G. de Lima ${ }^{\mathrm{b}}$ and V. Steffen Jr. ${ }^{\mathrm{c}, *}$ \\ ${ }^{a}$ Federal University of Tocantins, Mathematic Department Campus Arraias, Av. Universitária, Centro, CEP \\ 77330-000, Arraias-TO, Brazil \\ ${ }^{\mathrm{b}}$ Federal University of Itajubá, Mechanical Engineering Institute Campus José Rodrigues Seabra, P.O.Box 50, \\ CEP 37500-903, Itajubá-MG, Brazil \\ ${ }^{\mathrm{c}}$ Federal University of Uberlândia, School of Mechanical Engineering Campus Santa M ônica, P.O.Box 593, CEP \\ 38400-902, Uberlândia-MG, Brazil
}

\begin{abstract}
Dynamic vibration absorbers are discrete devices developed in the beginning of the last century used to attenuate the vibrations of different engineering structures. They have been used in several engineering applications, such as ships, power lines, aeronautic structures, civil engineering constructions subjected to seismic induced excitations, compressor systems, etc. However, in the context of nonlinear dynamics, few works have been proposed regarding the robust optimal design of nonlinear dynamic vibration absorbers. In this paper, a robust optimization strategy combined with sensitivity analysis of systems incorporating nonlinear dynamic vibration absorbers is proposed. Although sensitivity analysis is a well known numerical technique, the main contribution intended for this study is its extension to nonlinear systems. Due to the numerical procedure used to solve the nonlinear equations, the sensitivities addressed herein are computed from the first-order finite-difference approximations. With the aim of increasing the efficiency of the nonlinear dynamic absorber into a frequency band of interest, and to augment the robustness of the optimal design, a robust optimization strategy combined with the previous sensitivities is addressed. After presenting the underlying theoretical foundations, the proposed robust design methodology is performed for a two degree-of-freedom system incorporating a nonlinear dynamic vibration absorber. Based on the obtained results, the usefulness of the proposed methodology is highlighted.
\end{abstract}

Keywords: Nonlinear dynamics, dynamic vibration absorber, robust optimization, sensitivity analysis

\section{Introduction}

The use of discrete dynamic vibration absorbers (DVAs) in the problem of vibration attenuation constitutes an important subject in modern Engineering. The application of such systems to reduce noise and vibration levels in various types of engineering systems such as compressors systems, robots, ships, power lines, airplanes, helicopters, space structures, buildings and towers, etc., has been intensively investigated in the last decades. Much of the knowledge available to date is compiled in the original patent by Frahm [1], in the books by Den Hartog [2] and Koronev and Reznikov [3], and in some papers such as those by Steffen and Rade [4,5].

In the last decades, a great deal of effort has been devoted to the development of mathematical models for characterizing the nonlinear mechanical behavior of nonlinear dynamic vibration absorbers (nDVAs) accounting for their typical dependence on the parameters that are responsible for the nonlinearities. Besides the well-known

\footnotetext{
*Corresponding author. E-mail: vsteffen @ mecanica.ufu.br.
} 


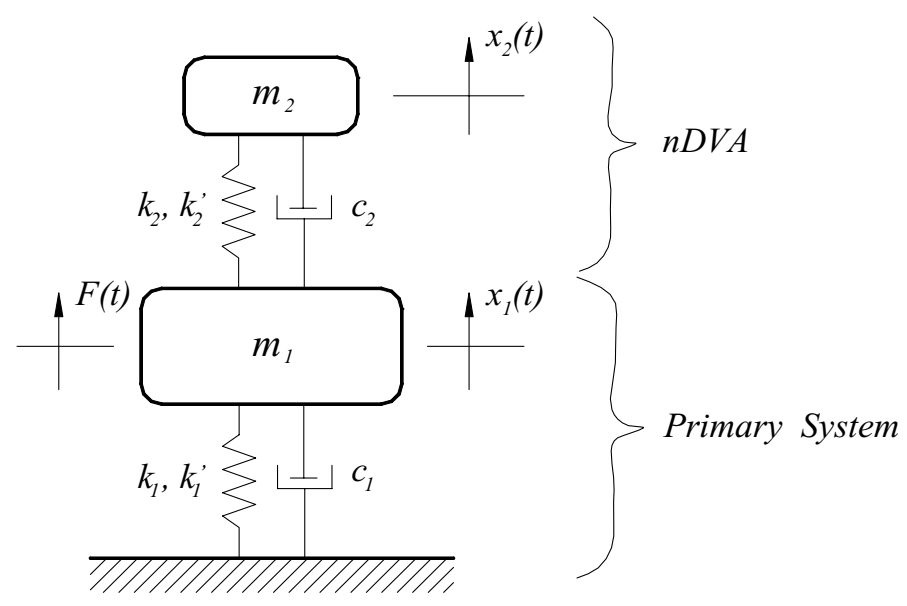

Fig. 1. Two degree-of-freedom mechanical system incorporating nDVA.

complexity of the modeling strategy involved in nonlinear dynamics, which constitutes a simple and straightforward means of representing the dynamic behavior of nDVAs, some methodologies have been suggested and have been shown to be particularly suitable to be used in combination with structural system discretization. Among these strategies, it should be mentioned the theoretical study proposed by Pipes [6] and by Pai and Schulz [7], in which techniques to improve the stability and efficiency of nDVAs into a frequency band of interest have been proposed. Rice and McCraith [8] suggested optimization strategies of systems incorporating nDVAs by applying an asymmetric nonlinear Duffing-type element incorporated in the suspension system of narrow-band absorption devices. Other works, such as those by Zang et al. [9] and Fonseca et al. [10] have considered the robust design strategies and its application into dynamics.

A straightforward extension of the modeling capability is the optimization of nDVA devices aiming at reducing the cost and/or maximizing their performance. In the quest for optimization, engineers are frequently faced with conflicting objectives, which naturally lead to the use of multicriteria optimization procedures [11]. In this context, an important topic to be addressed is the so-called sensitivity analysis, which enables to evaluate the degree of influence of variations of physical and/or geometrical parameters on the dynamic behavior of nDVAs. Sensitivity analysis constitutes an important step in various types of problems such as model updating, analysis of modified structures, optimal design, system identification, control and stochastic reliability assessment [12]. Several approaches have been developed for performing sensitivity analysis of dynamic responses, as reported in references [12,13]. However, applications to the case of structural systems containing nDVAs components combining with robust optimization strategies $[14,15]$ are not numerous, which motivates the study reported here.

In the following sections, the theoretical foundations related to various aspects regarding the strategy for modeling nDVAs, the sensitivity analysis, and the robust optimization procedure are first summarized, followed by the description of a numerical application that demonstrates its effectiveness when applied to the robust optimal design of structural systems incorporating nDVAs.

\section{Mathematical modeling of systems incorporating nDVAs.}

In this section, the mathematical modeling of systems incorporating nDVAs is summarized based on the original developments made by Natsiavas [16]. Figure 1 depicts the two degree-of-freedom (DOF) mechanical system incorporating a nDVA. The nonlinear system consists of a damped primary structure attached to the ground by a suspension including either a linear or a nonlinear spring, and a damped secondary mass coupled to the primary structure by a spring having nonlinear characteristics.

The interest is focused on frequency domain responses. In this case, to calculate the steady-state harmonic responses in the frequency domain, it is assumed the following relation for the external force, $f(t)=f_{0} \cos (\omega t)$, where $\omega$ is the excitation frequency. The constitutive forces of the springs are given as follows: 


$$
s_{i}\left(x_{i}\right)=k_{i} x_{i}+k_{i}^{\prime} x_{i}^{3}, \quad i=1,2
$$

where $x_{1}$ and $x_{2}$ represent, respectively, the displacement of the primary system with respect to the ground and the displacement of the nDVA with respect to the primary mass. In the present case, the dampers are linear and springs have nonlinear characteristics, where $k_{i}$ and $k_{i}^{\prime}$ indicate, respectively, their linear and nonlinear coefficients. By applying the Newton's second law, the following equations of motion of the nonlinear system is obtained:

$$
\begin{aligned}
& \left(m_{1}+m_{2}\right) \ddot{x}_{1}+m_{2} \ddot{x}_{2}+c_{1} \dot{x}_{1}+k_{1} x_{1}=f_{0} \cos (\omega t)-k_{1}^{\prime} x_{1}^{3} \\
& m_{2} \ddot{x}_{1}+m_{2} \ddot{x}_{2}+c_{2} \dot{x}_{2}+k_{2} x_{2}=-k_{2}^{\prime} x_{2}^{3}
\end{aligned}
$$

With the aim of obtaining the dimensionless normalized equations of motion for the nonlinear dynamic system, the displacements are normalized according to the following relation $y_{i}=x_{i} / x_{c}$, where $x_{c}=f_{0} / k_{1}$ and $i=1,2$. In addition, the following relations are introduced: $\zeta_{1}=c_{1} /\left(2 \sqrt{k_{1} m_{1}}\right), \zeta_{2}=c_{2} /\left(2 \sqrt{k_{2} m_{2}}\right), \varepsilon_{1}=k_{1}^{n l} x_{c}^{2} /\left(m_{1} \omega^{2}\right)$, $\varepsilon_{2}=k_{2}^{n l} x_{c}^{2} /\left(m_{2} \omega^{2}\right), \mu=m_{2} / m_{1}, F=f_{0} /\left(m_{1} x_{c} \omega^{2}\right), \bar{\omega}_{1}^{2}=k_{1} / m_{1}, \bar{\omega}_{2}^{2}=k_{2} / m_{2}, \omega_{1}=\bar{\omega}_{1} / \omega$, and $\omega_{2}=\bar{\omega}_{2} / \omega$. After manipulations, the following matrix form of the normalized equations is obtained:

$$
\mathbf{M} \ddot{\mathbf{y}}(t)+\mathbf{C} \dot{\mathbf{y}}(t)+\mathbf{K y}(t)=F(t)
$$

where the normalized mass, damping and stiffness matrices are expressed, respectively, as:

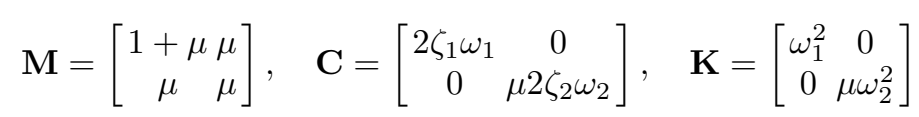

and the normalized displacement and force vectors are given, respectively, as follows:

$$
y(t)=\left\{\begin{array}{l}
y_{1}(t) \\
y_{2}(t)
\end{array}\right\}, \quad F(t)=\left\{\begin{array}{l}
F \cos (\omega t)-\varepsilon_{1} y_{1}^{3} \\
-\mu \varepsilon_{2} y_{2}^{3}
\end{array}\right\}
$$

\subsection{Steady-state harmonic responses of the nonlinear dynamic system.}

Various perturbation methods are based on averaging. This means that the unknown functions of the problem are considered as dependent variables by using a shift of variables from the original dependent variable [17,18]. These methods encompass techniques such as the following [19]: Krylov-Bogoliubov method, Krylov-BogoliubovMitropolsky method, and the method of the generalized average. In the present case, the Krylov-Bogoliubov method will be used to integrate Eq. (3), leading to an approximate solution of the nonlinear differential equations of motion. Within this context, the Van der Pol Transformation [20], represented by expressions Eqs (6) and (7), are used to guarantee that the transformation is unique.

$$
\begin{aligned}
& \mathbf{y}(\tau)=\mathbf{u}(\tau) \cos \tau+\mathbf{v}(\tau) \sin \tau \\
& \dot{\mathbf{y}}(\tau)=-\mathbf{u}(\tau) \sin \tau+\mathbf{v}(\tau) \cos \tau
\end{aligned}
$$

where $\mathbf{u}=\left(u_{1}, u_{2}\right)^{\mathrm{T}}$ and $v=\left(v_{1}, v_{2}\right)^{\mathrm{T}}$ are assumed to be slow functions of the normalized time $\tau=\omega t$. The differentiation of relations Eqs (6) and (7) with respect to $\tau$ leads to the following expressions:

$$
\begin{aligned}
& \dot{\mathbf{y}}(\tau)=-\dot{\mathbf{u}}(\tau) \cos \tau-\mathbf{u}(\tau) \sin \tau+\dot{\mathbf{v}}(\tau) \sin \tau+\mathbf{v}(\tau) \cos \tau \\
& \ddot{\mathbf{y}}(\tau)=-\dot{\mathbf{u}}(\tau) \sin \tau-\mathbf{u}(\tau) \cos \tau+\dot{\mathbf{v}}(\tau) \cos \tau+\mathbf{v}(\tau) \sin \tau
\end{aligned}
$$

Then, by taking into account the relation Eqs (7), (8) assumes the following form:

$$
\dot{\mathbf{u}}(\tau) \cos \tau+\dot{\mathbf{v}}(\tau) \sin \tau=0
$$

Substituting expressions Eqs (6), (7) and (9) into the Eq. (3), yields:

$$
(\mathbf{M} \dot{\mathbf{v}}-\mathbf{M u}+\mathbf{C v}+\mathbf{K u}) \cos \tau-(\mathbf{M} \dot{\mathbf{u}}+\mathbf{M v}+\mathbf{C u}-\mathbf{K} \mathbf{v}) \sin \tau=F(\mathbf{u}, \mathbf{v}, \tau)
$$


Next, Eq. (10) is multiplied by $\mathbf{M} \cos \tau$ and Eq. (11) is multiplied by $-\sin \tau$, and after that the resulting equations must be added up and integrated over the period ( 0 to $2 \pi$ ), in which $\mathbf{u}$ and $\mathbf{v}$ are taken as constants along this period (it represents a very short time interval [21]). After algebraic manipulations one obtains the following expression:

$$
\mathbf{M} \dot{\mathbf{u}}=\frac{1}{2}(\mathbf{K}-\mathbf{M}) \mathbf{v}-\frac{1}{2}\left\{\begin{array}{c}
2 \zeta_{1} \omega_{1} u_{1}-\frac{3}{4} \varepsilon_{1} v_{1}\left(u_{1}^{2}+v_{1}^{2}\right) \\
\mu 2 \zeta_{2} \omega_{2} u_{2}-\frac{3}{4} \mu \varepsilon_{2} v_{2}\left(u_{2}^{2}+v_{2}^{2}\right)
\end{array}\right\}
$$

Similarly, Eqs (10) and (11) must be multiplied by $\mathbf{M} \sin \tau$ and $\cos \tau$, respectively, and then added up, so that the resulting equation must be integrated over the same period as above, and leads to the following expression:

$$
\mathbf{M} \dot{\mathbf{v}}=\frac{1}{2}(\mathbf{M}-\mathbf{K}) u-\frac{1}{2}\left\{\begin{array}{c}
-F+2 \zeta_{1} \omega_{1} v_{1}+\frac{3}{4} \varepsilon_{1} u_{1}\left(u_{1}^{2}+v_{1}^{2}\right) \\
\mu 2 \zeta_{2} \omega_{2} v_{2}+\frac{3}{4} \mu \varepsilon_{2} u_{2}\left(u_{2}^{2}+v_{2}^{2}\right)
\end{array}\right\}
$$

Equations (12) and (13) represent a first order ordinary differential system of equations with the following four variables $\left(u_{1}, u_{2}, v_{1}, v_{2}\right)$. The solution originated from the averaging method corresponds to motions of period $2 \pi$ for the original system, Eq. (3). In the case of steady-state periodic vibrations, the following condition is used:

$$
\dot{\mathbf{u}}=\dot{\mathbf{v}}=0
$$

By substituting Eq. (14) into Eqs (12) and (13), the following nonlinear algebraic system composed by four equations and four variables $\left(u_{1}, u_{2}, v_{1}, v_{2}\right)$ is obtained:

$$
\left\{\begin{array}{l}
\left(1+\mu-\omega_{1}^{2}\right) u_{1}+\mu u_{2}-2 \zeta_{1} \omega_{1} v_{1}-\frac{3}{4} \varepsilon_{1} u_{1}\left(u_{1}^{2}+v_{1}^{2}\right)+F=0 \\
\mu u_{1}+\left(\mu-\mu \omega_{2}^{2}\right) u_{2}-\mu 2 \zeta_{2} \omega_{2} v_{2}-\frac{3}{4} \mu \varepsilon_{2} u_{2}\left(u_{2}^{2}+v_{2}^{2}\right)=0 \\
\left(\omega_{1}^{2}-1-\mu\right) v_{1}-\mu v_{2}-2 \zeta_{1} \omega_{1} u_{1}+\frac{3}{4} \varepsilon_{1} v_{1}\left(u_{1}^{2}+v_{1}^{2}\right)=0 \\
\mu v_{1}+\left(\mu-\mu \omega_{2}^{2}\right) v_{2}+\mu 2 \zeta_{2} \omega_{2} u_{2}-\frac{3}{4} \mu \varepsilon_{2} v_{2}\left(u_{2}^{2}+v_{2}^{2}\right)=0
\end{array}\right.
$$

The system of Eq. (15) can be numerically solved by using the function "fsolve" from MATLAB ${ }^{\circledR}$ toolbox [22]. Then, the obtained values of the parameters $\left(u_{1}, u_{2}, v_{1}, v_{2}\right)$ are used to calculate the amplitudes of vibrations of the nonlinear system shown in Fig. 1, by performing the following relations $r_{1}=\sqrt{u_{1}^{2}+v_{1}^{2}}$ (for the primary mass) and $r_{2}=\sqrt{u_{2}^{2}+v_{2}^{2}}$ (for the nDVA). Moreover, in the numerical computation, the parameters of force and frequency are also considered: $\beta=F / \omega_{1}^{2}$ (force parameter) and $\Omega=\omega / \bar{\omega}_{1}$ (frequency parameter), so that in Eq. (15), $\omega_{1}=1 / \Omega$ and $\omega_{2}=\rho / \Omega$, where $\rho=\omega_{2} / \omega_{1}$ (frequency ratio).

\section{Finite-difference approach to sensitivity analysis of dynamic responses}

The global matrices appearing in Eq. (3) establish the dependence of the response of the system with respect to a set of design parameters. Such functional dependence can be expressed in a general form as follows:

$$
r=r[M(p), C(p), K(p)]
$$

where $r$ and $p$ designate vectors of structural responses and design parameters, respectively.

The sensitivity of the responses with respect to a given parameter $p_{i}$, evaluated for a given set of values of the design parameters $p^{0}$, is defined as the following partial derivative:

$$
\left.\frac{\partial r}{\partial p_{i}}\right|_{p^{0}}=\lim _{\Delta p_{i} \rightarrow 0}\left\{\frac{r\left[M\left(p_{i}^{0}+\Delta p_{i}\right), C\left(p_{i}^{0}+\Delta p_{i}\right), K\left(p_{i}^{0}+\Delta p_{i}\right)\right]}{\Delta p_{i}}-\frac{r\left[M\left(p_{i}^{0}\right), C\left(p_{i}^{0}\right), K\left(p_{i}^{0}\right)\right]}{\Delta p_{i}}\right\}
$$

where $\Delta p_{i}$ is a small arbitrary variation, applied to the current value of the parameter $p_{i}^{0}$, while all other parameters are kept unchanged. The sensitivity of the response with respect to $p_{i}$ can be numerically estimated by using 
finite differences by successively computing the responses corresponding to $p_{i}=p_{i}^{0}$ and $p_{i}=p_{i}^{0}+\Delta p_{i}$, and then calculating:

$$
\left.\frac{\partial r}{\partial p_{i}}\right|_{p^{0}} \approx\left\{\frac{r\left[M\left(p_{i}^{0}+\Delta p_{i}\right), C\left(p_{i}^{0}+\Delta p_{i}\right), K\left(p_{i}^{0}+\Delta p_{i}\right)\right]}{\Delta p_{i}}-\frac{r\left[M\left(p_{i}^{0}\right), C\left(p_{i}^{0}\right), K\left(p_{i}^{0}\right)\right]}{\Delta p_{i}}\right\}
$$

Such a procedure is an estimated approach enabling the calculation of the sensitivity of the dynamic responses of the nonlinear system with respect to the small modifications introduced in the design parameters. Moreover, the accuracy of the sensitivity estimates depends upon the choice of the value of the parameter increment $\Delta p_{i}$, which has to be small as compared to the corresponding parameters $p_{i}$. Contradictorily, numerical instabilities are prone to appear as $\Delta p_{i}$ is made close to zero. Another strategy consists in computing the analytical derivatives, if possible, of the structural responses with respect to the parameters considered [12]. As numerical procedures are used to solve the nonlinear system of Eq. (15), this approach is not considered in the present case.

\section{Stochastic multiobjective optimization problem}

A general multiobjective optimization problem (MOP) involves the simultaneous optimization of multiple objective functions, which may be in conflict with each other. The goal is to find the best design solutions, which lead to the minimum or maximum values of the various objective functions considered. In general, a multiobjective optimization problem has no single optimal solution and the interaction among different objectives gives rise to a set of compromised solutions, known as Pareto optimal solutions [11]. Since none of these Pareto optimal solutions can be identified as better than the others without any further consideration, the issue here is to find as many Pareto optimal solutions as possible. A deterministic multiobjective problem includes a set of $k$ parameters (decision variables) and a set of $n$ objective functions $(n \geqslant 2)$ that can be summarized as follows:

$$
\left\{\begin{array}{l}
\min _{\mathbf{x}} F(\mathbf{x})=\left[f_{1}(\mathbf{x}), f_{2}(\mathbf{x}), \ldots, f_{n}(\mathbf{x})\right] \\
g_{j}(\mathbf{x}) \leqslant 0 \quad j=1, \ldots, m \text { and } \mathbf{x}_{L} \leqslant \mathbf{x} \leqslant \mathbf{x}_{U} \quad \mathbf{x} \in C
\end{array}\right.
$$

where $\mathbf{x}=\left[x_{1}, x_{2}, \ldots, x_{k}\right]^{T}$ is a vector of design variables; $C \subset R^{k}$ is the design space associated to the equality or inequality constraints represented by $g_{j}(\mathbf{x})$. In practical design environment the vector $F(\mathbf{x})$ is non-linear, multi-modal and not analytically expressed.

In the classical deterministic optimization process, the uncertainties of the design variables are not taken into account. Consequently, the Pareto optimal solutions are not insensitive to the variations on the design parameters. Regarding the robustness evaluation of the optimal solutions, the problem posed is not to find only the optimum but the robust optimum by taking into account the uncertainties found along the optimization process. In nonlinear dynamics, uncertainties result from several sources, including the errors associated to the modeling of nonlinear physical phenomena, the materials characteristics adopted (such as Young's modulus, mass density, Poisson ratio, etc.), the geometry characteristics, the tolerances in the manufacturing processes (thickness, stiffness of junctions, boundary conditions, etc.). Classically, the robustness of the optimal solution is evaluated starting from the deterministic optimization process, supposing that the deterministic design space contains the robust solutions obtained through the inclusion of stochastic criteria.

To mitigate the difficulty in evaluating the robustness of optimal solutions, some authors such as those in [15] proposed an optimization methodology through which an additional objective function is defined, having the same mean and standard deviation as the original objective function, by using a weighting objective method. The main disadvantage of the proposed methodology is that not all the Pareto optimal solutions can be found, unless the problem is convex. The authors in reference [14] proposed a similar procedure by using the Taguchi method, in which the robustness of the optimal solutions is evaluated only at the end of the optimization process. In this paper, a different methodology to evaluate the robustness of the optimal solutions is drawn by supposing that an optimal and robust solution is not necessarily a solution included in the deterministic design space. This can be done by considering that the robustness function defined by Eq. (20) is introduced as an additional objective function into the original multiobjective optimization problem. 


$$
f^{r}=\left(\sigma_{f} / \mu_{f}\right)=\left(f^{v}\right)^{-1}
$$

where $f^{v}=\sigma_{f} / \mu_{f}$ is the vulnerability of the cost function $f, \mu_{f}$ and $\sigma_{f}$ are, respectively, the mean and the standard deviation that are computed by taking into account the uncertainties on the design variables. To introduce the uncertainties on the design variables, the so-called Latin-Hyper-Cube (LHC) sampling method [23] is used with the aim of evaluating the dispersion of each original cost function taken into account during the optimization process. To evaluate the uncertainties on the design variables, one considers the parametric approach, by assuming that all design variables exhibit normal distributions with zero mean and unit variance.

The stochastic optimization is performed by using the Non-dominated Sorting Genetic Algorithm (NSGA) method, originally developed by Srinivas and Deb [24], in which a selection operator is used to classify the nondominated individuals. In the stochastic optimization, the robustness function associated with a cost function is introduced as an additional cost function that must be maximized simultaneously with the original cost functions. In this case, the initial multiobjective optimization problem composed by $n$ cost functions is transformed into a stochastic optimization problem with $2 n$ objective functions.

\section{Numerical results}

The following numerical example is presented to illustrate the application of the proposed methodology in order to obtain a robust design of the nonlinear dynamic vibration absorber shown in Fig. 1. The nominal values of the design parameters used to generate the dynamic responses of the nonlinear system are presented in Table 1 . The computation consist in obtaining the driving point dynamic responses $H(\omega, p)$ associated to the displacement $x_{1}$ indicated on Fig. 1, in the frequency band of interest $\Omega=[0.7-1.3 \mathrm{~Hz}]$ comprising a total number of 300 frequency points. Moreover, for the numerical resolution of the Eq. (15) the following initial conditions have been adopted $\left(u_{1}=5, u_{2}=5, v_{1}=2, v_{2}=4\right)$.

Table 1

Nominal values of the design variables

\begin{tabular}{cccccccc}
\hline Parameters & $\varepsilon_{1}$ & $\varepsilon_{2}$ & $\zeta_{1}$ & $\zeta_{2}$ & $\mu$ & $\beta$ & $\rho$ \\
\hline Nominal values & $1 \times 10^{-4}$ & 0.01 & 0.01 & 0.01 & 0.05 & 0.1 & 1.0 \\
\hline
\end{tabular}

\subsection{Sensitivities of the dynamic responses with respect to structural parameters}

In this example, the normalized structural parameters depicted on Table 1 are considered as the design variables in the computation of the normalized sensitivities of the dynamic responses with respect to a given parameter $p_{i}$, $S^{N}\left(\omega, p_{i}\right)$. The normalized parts of the approximated sensitivity functions calculated by finite differences according to Eq. (18) are shown in Figs 2 to 8, for which differents successively larger variations of the design parameters, corresponding to $1 \%, 5 \%, 10 \%$ and $20 \%$ of the nominal values, respectively, were adopted. Also, in the same figures, the real parts of the dynamic responses $H(\omega, p)$, multiplied by convenient scale factors (SF), are shown. The sensitivity functions have been normalized according to the following scheme:

$$
S^{N}\left(\omega, p_{i}\right)=\frac{H\left(\omega, p_{i}\right)-H\left(\omega, p_{0}\right)}{\Delta p_{i}} \frac{p_{0}}{H\left(\omega, p_{0}\right)}
$$

The most immediate use of the sensitivity functions is the qualitative comparison of the level degrees of influence of different design parameters on the dynamic responses: the larger the amplitude of the sensitivity function with respect to a given parameter, the larger the influence of this parameter on both the amplitude of the harmonic response and the suppression bandwidth (see Fig. 9). Clearly, it should be pointed out that this reasoning is strictly valid for small variations of the design parameters because, depending on the case, approximations of finite response variations can provide better results. In addition, the sensitivity analysis enables to decide among the design parameters those that should be retained in the optimization process of the nDVA. It should be noted that by analyzing the sensitivities of the parameters, it is possible to observe that both the parameters $\zeta_{1}$ and $\zeta_{2}$ do not have a significant influence 

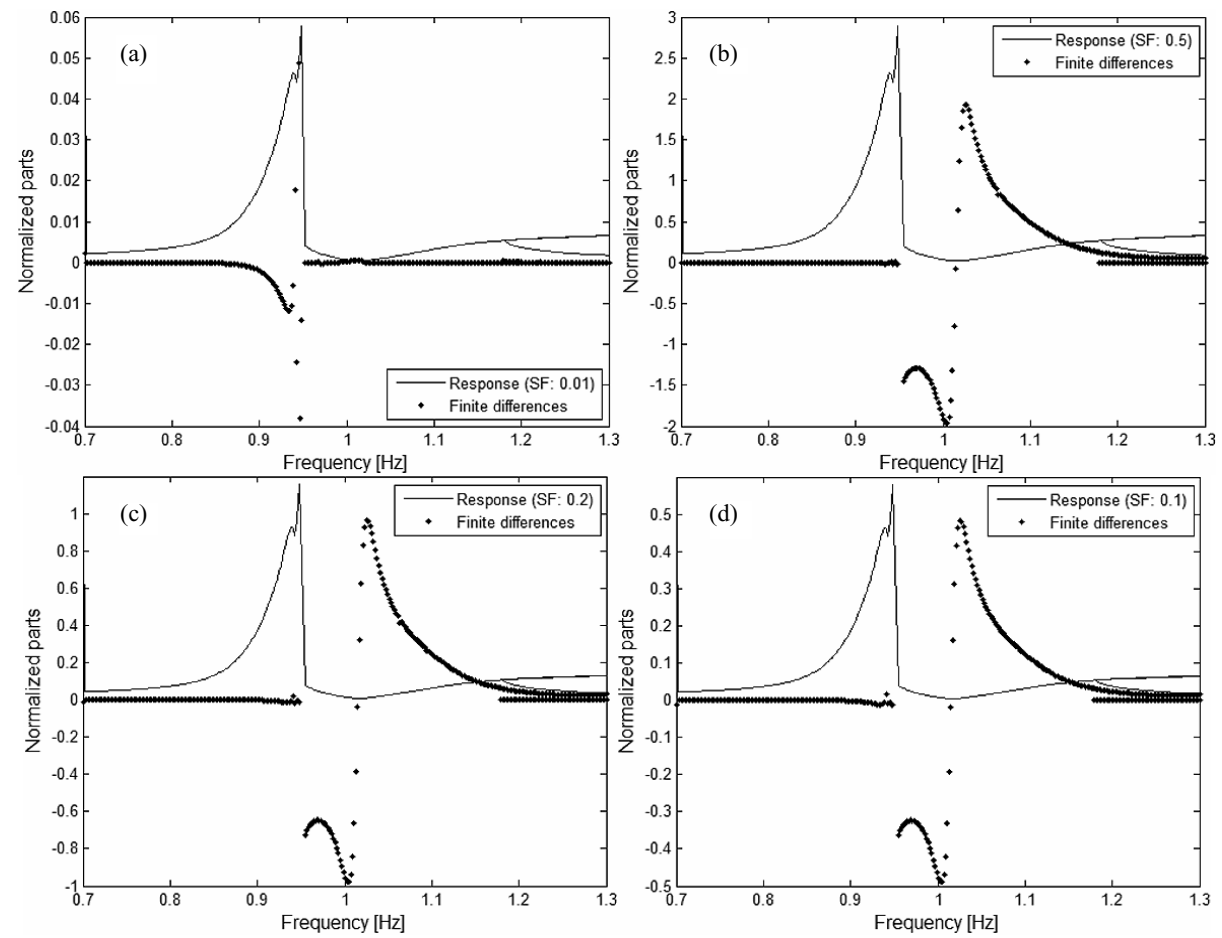

Fig. 2. Finite difference estimations of the Response $H(\omega, p)$ with respect to $\varepsilon_{1}$ : (a) $\Delta \varepsilon_{1} / \varepsilon_{1}=1 \%$; (b) $\Delta \varepsilon_{1} / \varepsilon_{1}=5 \%$; (c) $\Delta \varepsilon_{1} / \varepsilon_{1}=10 \%$; (d) $\Delta \varepsilon_{1} / \varepsilon_{1}=20 \%$.
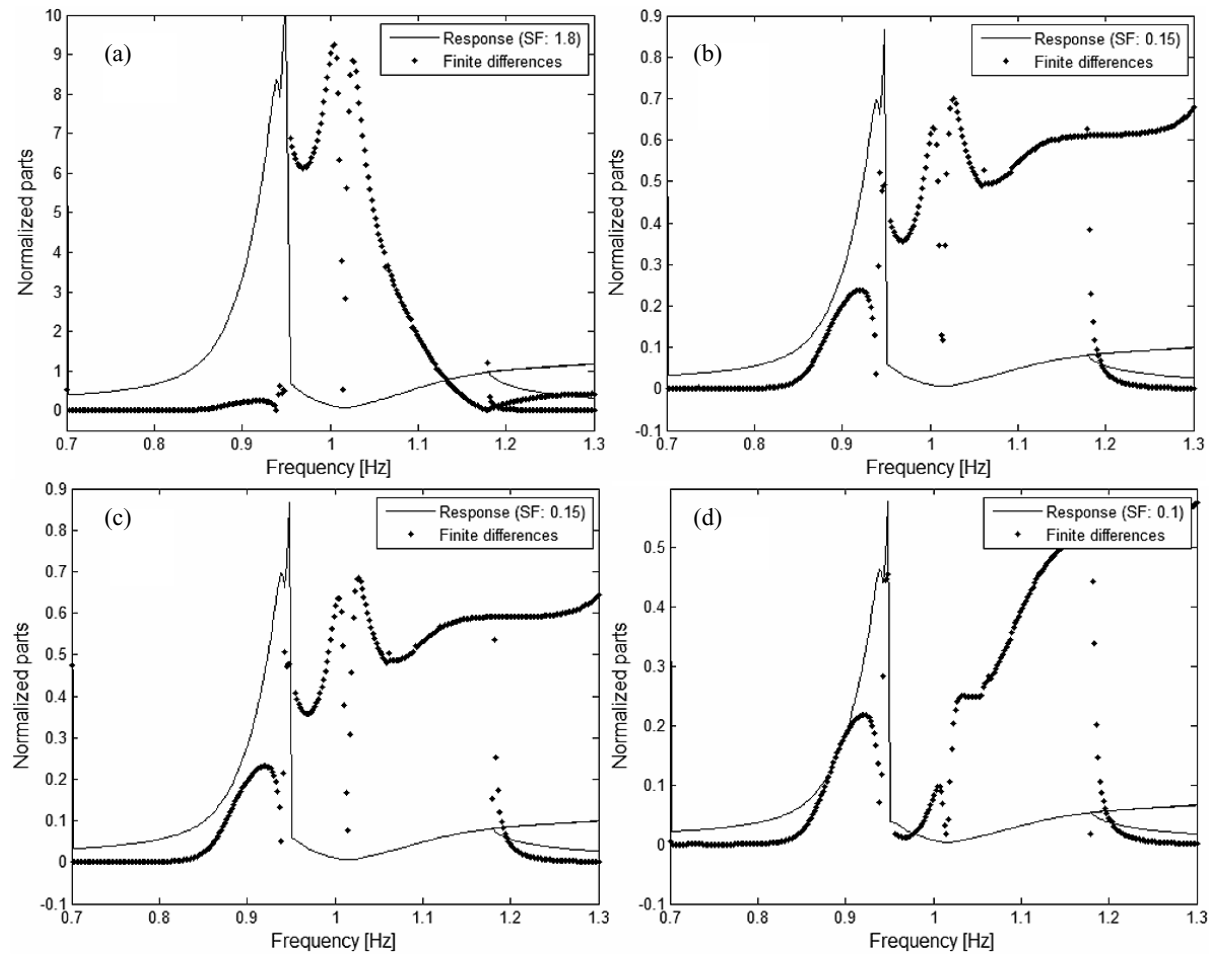

Fig. 3. Finite difference estimations of the Response $H(\omega, p)$ with respect to $\varepsilon_{2}$ : (a) $\Delta \varepsilon_{2} / \varepsilon_{2}=1 \%$; (b) $\Delta \varepsilon_{2} / \varepsilon_{2}=5 \%$; (c) $\Delta \varepsilon_{2} / \varepsilon_{2}=10 \%$; (d) $\Delta \varepsilon_{2} / \varepsilon_{2}=20 \%$. 

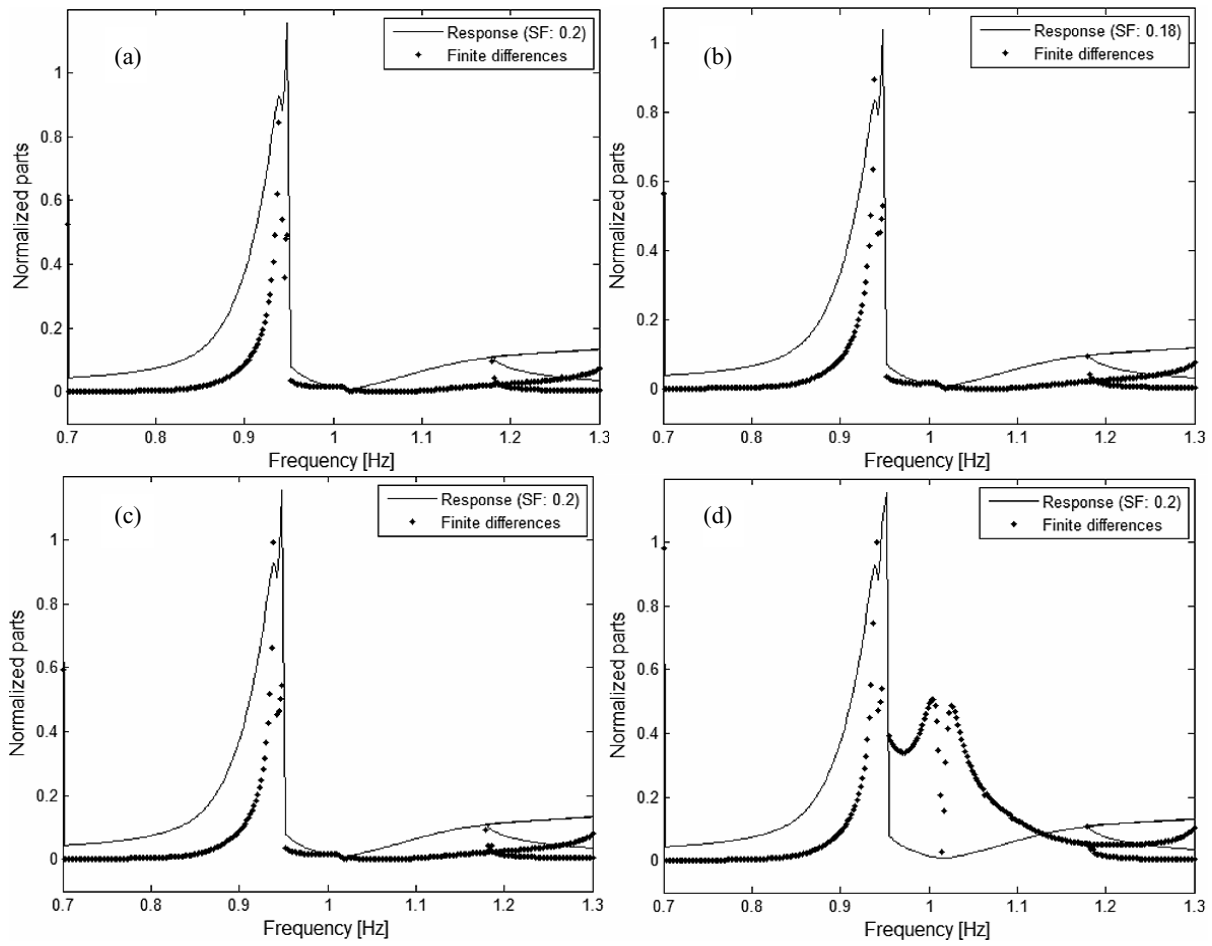

Fig. 4. Finite difference estimations of the Response $H(\omega, p)$ with respect to $\xi_{1}$ : (a) $\Delta \xi_{1} / \xi_{1}=1 \%$; (b) $\Delta \xi_{1} / \xi_{1}=5 \%$; (c) $\Delta \xi_{1} / \xi_{1}=10 \%$; (d) $\Delta \xi_{1} / \xi_{1}=20 \%$.
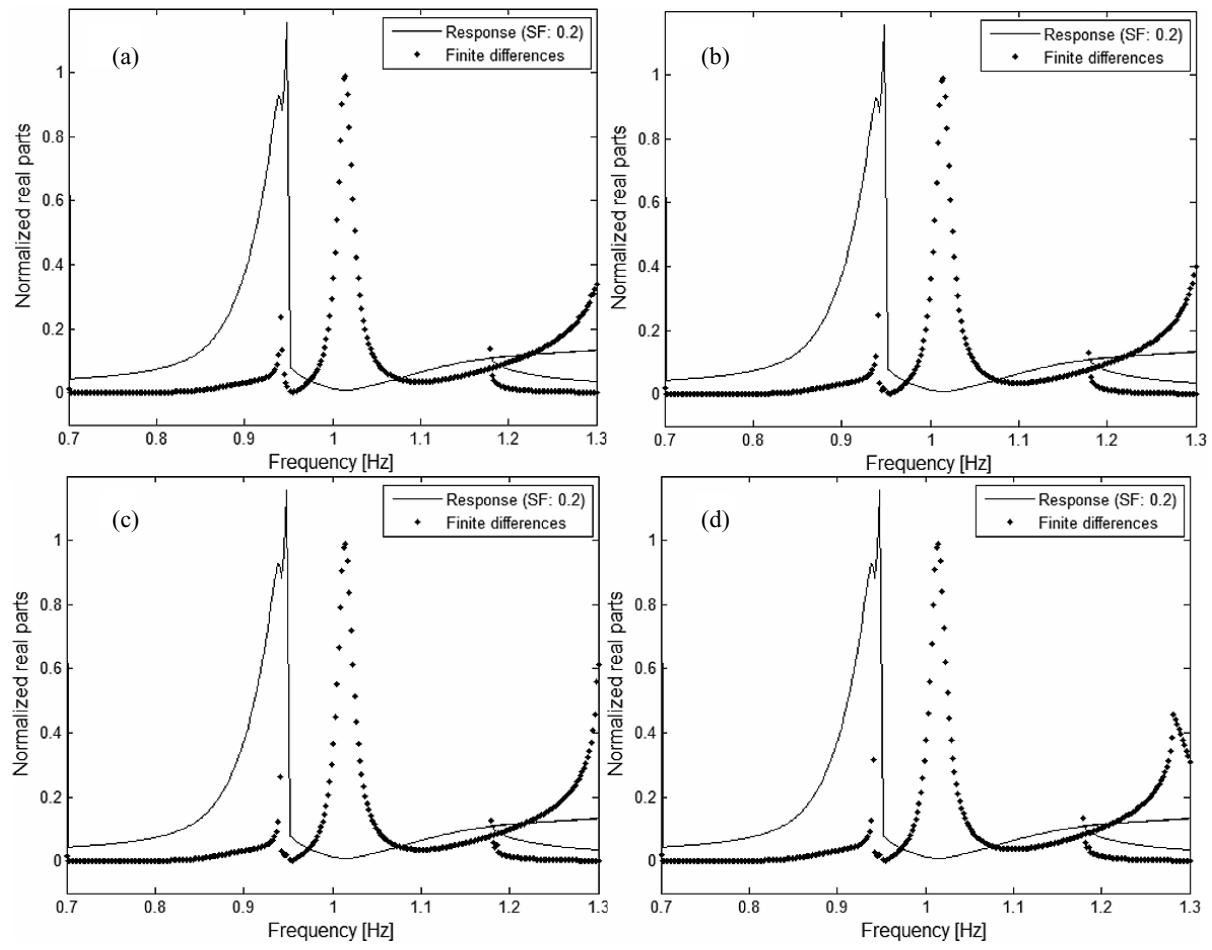

Fig. 5. Finite difference estimations of the Response $H(\omega, p)$ with respect to $\xi_{2}$ : (a) $\Delta \xi_{2} / \xi_{2}=1 \%$; (b) $\Delta \xi_{2} / \xi_{2}=5 \%$; (c) $\Delta \xi_{2} / \xi_{2}=10 \%$; (d) $\Delta \xi_{2} / \xi_{2}=20 \%$. 

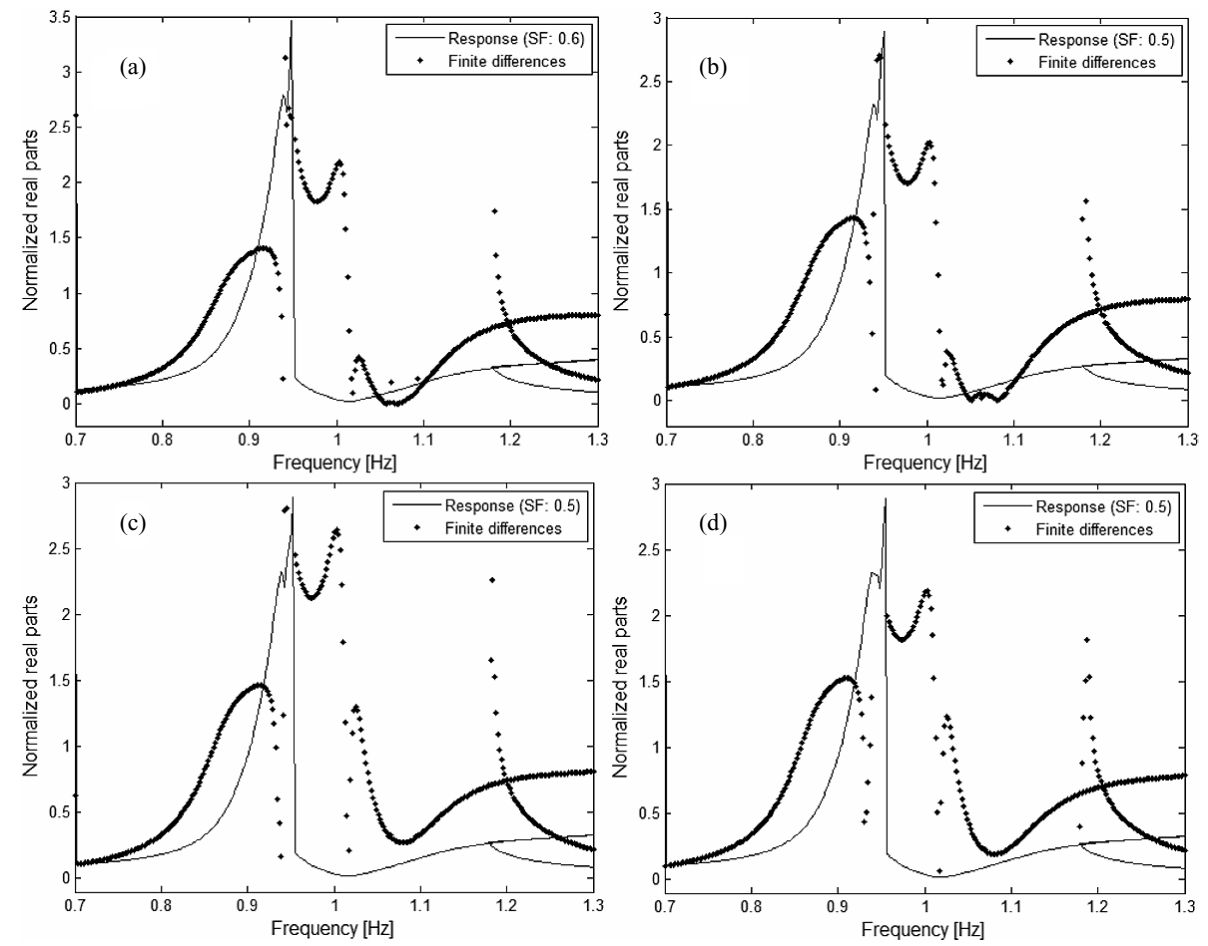

Fig. 6. Finite difference estimations of the Response $H(\omega, p)$ with respect to $\mu$ : (a) $\Delta \mu / \mu=1 \%$; (b) $\Delta \mu / \mu=5 \%$; (c) $\Delta \mu / \mu=10 \%$; (d) $\Delta \mu / \mu=20 \%$.
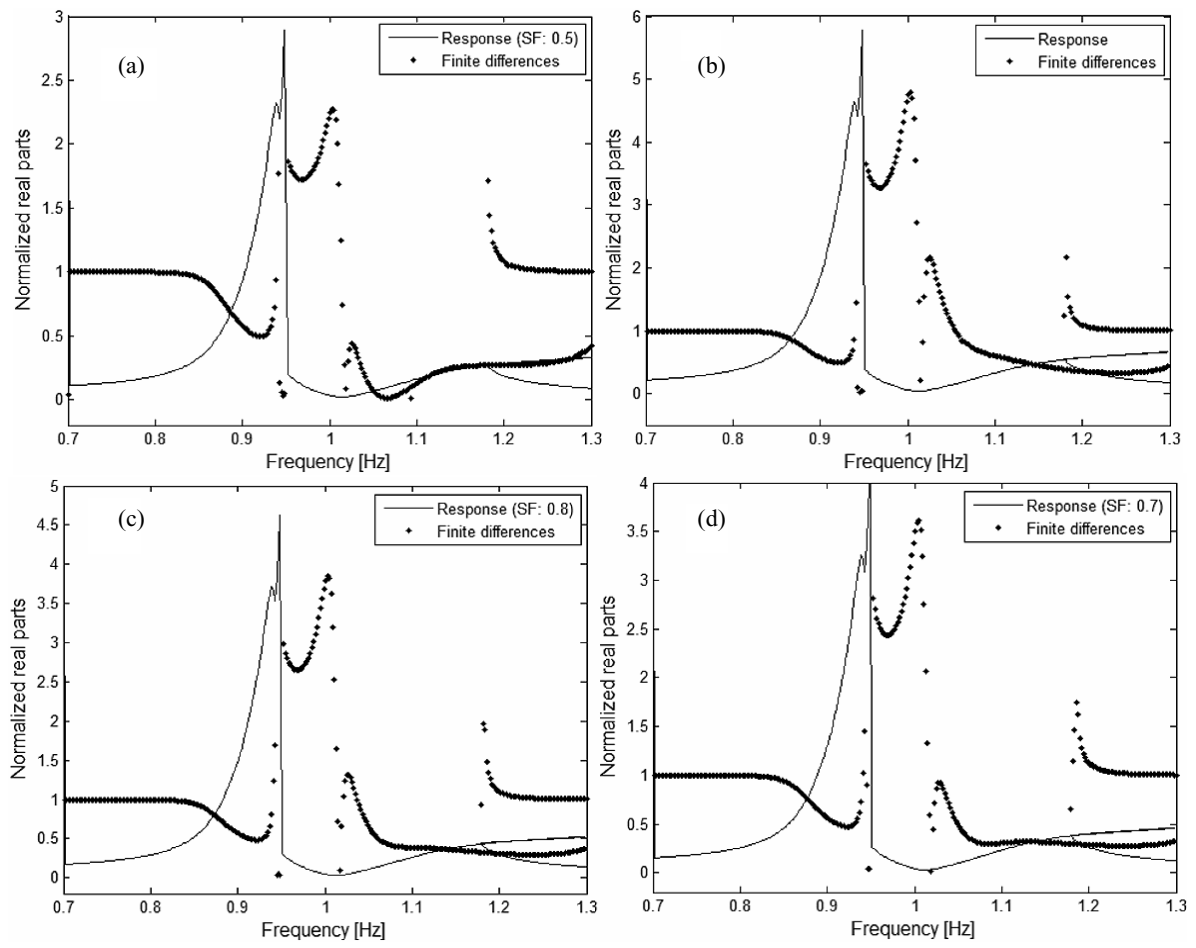

Fig. 7. Finite difference estimations of the Response $H(\omega, p)$ with respect to $\beta$ : (a) $\Delta \beta / \beta=1 \%$; (b) $\Delta \beta / \beta=5 \%$; (c) $\Delta \beta / \beta=10 \%$; (d) $\Delta \beta / \beta=20 \%$. 

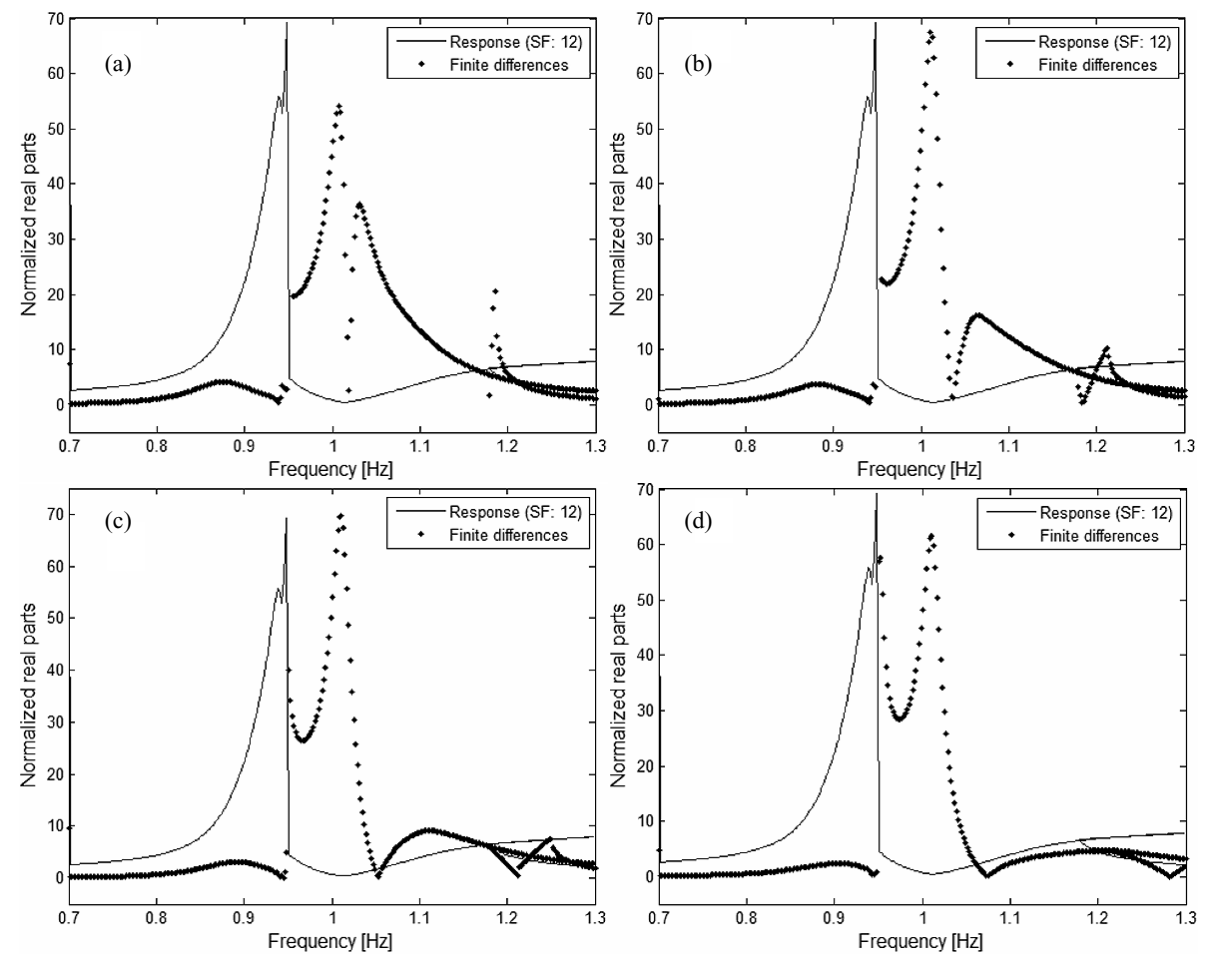

Fig. 8. Finite difference estimations of the Response $H(\omega, p)$ with respect to $\rho$ : (a) $\Delta \rho / \rho=1 \%$; (b) $\Delta \rho / \rho=5 \%$; (c) $\Delta \rho / \rho=10 \%$; (d) $\Delta \rho / \rho=20 \%$.

on the evaluation of the suppression bandwidth. Similarly, the sensitivity of the parameter $\varepsilon_{1}$ (nonlinear coefficient of the spring that connects the primary mass to the base) with respect to the suppression bandwidth is also small. Consequently, these parameters are not considered as design variables in the robust optimization process. However, the influence of the parameters $\varepsilon_{2}$ (nonlinear coefficient of the spring that connects the primary mass to the nDVA), $\beta$ (force parameter), $\mu$ (mass ratio), and $\rho$ (frequency ratio) both on the resonance peak and the suppression band are larger than those calculated for the previously considered variables. For this reason, these four parameters have been retained as design variables in the optimization process.

\subsection{Robust optimal design of $n D V A$}

After having verified the influence of each design variable on the dynamic response of the nonlinear system, the interest now is to evaluate the robust optimization strategy for the optimization of the nDVA depicted in Fig. 1. The deterministic optimization problem is composed by the following two objective functions: the first cost function is the amplitude of the dynamic response of the nonlinear damped system corresponding to the mode 1 (M1) (by minimizing the amplitude of the response at the corresponding resonance peak); the second cost function is the maximization of the suppression bandwidth. Figure 9 depicts the objective functions that are mathematically defined as follows:

$$
\text { minimize: }\left\{\begin{array}{l}
f_{1}=\text { amplitude }(M 1) \\
f_{2}=- \text { suppression bandwidth }
\end{array}\right.
$$

The design parameters and their corresponding admissible variations are illustrated on Table 2 . These ranges were chosen according to the sensitivity analysis presented in the previous section so that large variations with respect to the nominal values are avoided. Only the ranges of the continuous variables are taken as constraints in the robust optimization step. The stochastic optimization problem is composed by the defined objective functions, Eq. (22), and the following additional robustness functions associated with each cost function: 
Table 2

Design variables and their admissible variations and uncertainties

\begin{tabular}{cccc}
\hline Design parameters & Nominal values & Variations & Uncertainty level \\
\hline$\varepsilon_{2}$ & 0.01 & $\pm 30 \%$ & $\Delta \varepsilon_{2} / \varepsilon_{2}=3.0 \%$ \\
$\mu$ & 0.05 & $\pm 30 \%$ & $\Delta \mu / \mu=3.0 \%$ \\
$\beta$ & 0.1 & $\pm 30 \%$ & $\Delta \beta / \beta=3.0 \%$ \\
$\rho$ & 1.0 & $\pm 30 \%$ & $\Delta \rho / \rho=3.0 \%$ \\
\hline
\end{tabular}

Table 3

Optimal and robust solutions for points $P d$ and $P r$, respectively

\begin{tabular}{clccc}
\hline Optimal point & \multicolumn{1}{c}{$\varepsilon_{2}$} & $\mu$ & $\beta$ & $\rho$ \\
\hline $\mathrm{Pd}$ & 0.0091724 & 0.054959 & 0.090 & 1.10 \\
$\mathrm{Pr}$ & 0.010462 & 0.064458 & 0.095 & 1.25 \\
\hline
\end{tabular}

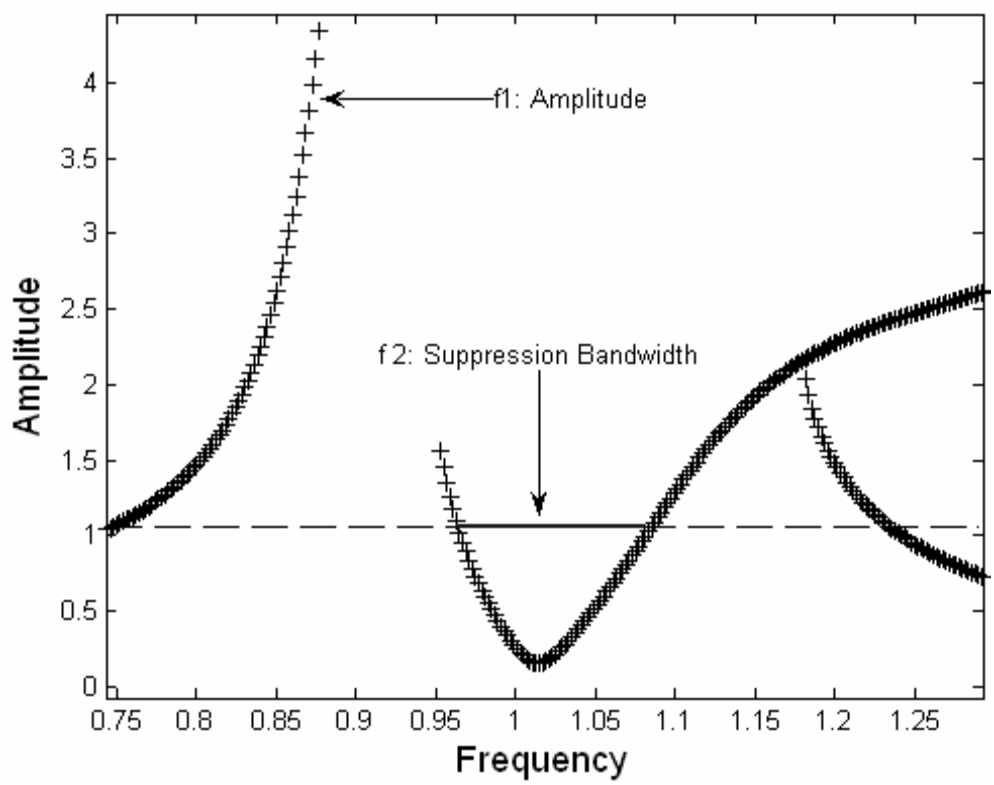

Fig. 9. Representation of the objective functions $f_{1}$ and $f_{2}$.

minimize: $F(x)=\left(f_{1}, f_{1}^{v}, f_{2}, f_{2}^{v}\right)$

where $f_{1}^{v}=\left(\sigma_{1} / \mu_{1}\right)^{-1}$ and $f_{2}^{v}=\left(\sigma_{2} / \mu_{2}\right)^{-1}$. The interest is to optimize the cost functions and to maximize the robustness functions, simultaneously.

The parameters of the NSGA are defined as follows: probability of selection, 0.25 ; probability of crossover, 0.25 ; probability of mutation, 0.25 ; number of generations, 100 ; number of individuals per generation, 30 ; and the sharing coefficient, 0.2 . To find the robust optimal solutions by using the NSGA algorithm, for each generation one introduces the uncertainties defined in Table 2 by performing the LHC sampling method. Within this context 1,000 samples for each design variable will be evaluated to generate the vulnerability function of each cost function in terms of the level of dispersion.

Figure 10 shows the NSGA solutions obtained by applying the proposed robust method, representing the cost functions and their vulnerabilities. In practice, the vulnerability functions consist in minimizing the dispersion around each cost function. Through this figure, one can notice that the intervals of dispersion for the cost functions are as follows: from 0.006 to 0.014 for the optimal solutions corresponding to the first cost function, and from approximately 0 to 0.05 for the optimal solutions corresponding to the second cost function.

Figure 11 enables to compare the robust solutions and the deterministic solutions. One can conclude that the deterministic solutions exhibit a better performance than the robust solutions. However, obviously the deterministic 

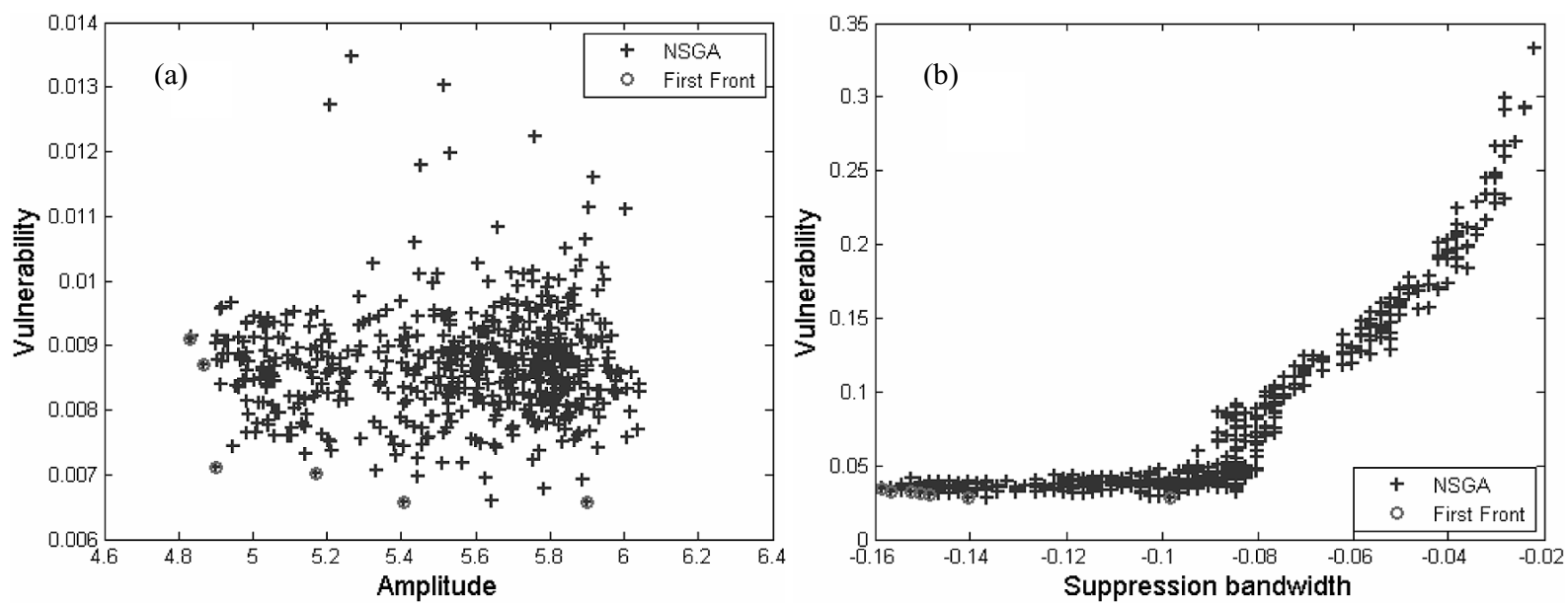

Fig. 10. NSGA solutions and the first front of Pareto for both objective and vulnerability functions.

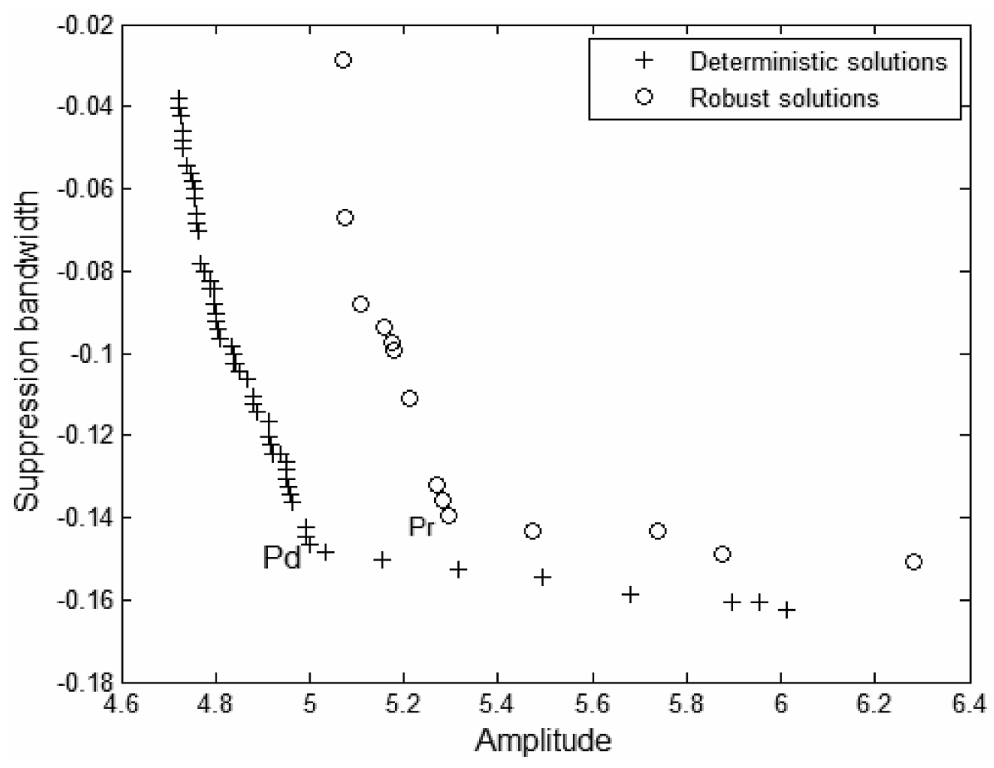

Fig. 11. Comparison between the deterministic solutions and the robust solutions.

solutions are not robust with respect to uncertainties on the design parameters.

For the deterministic and robust set of solutions corresponding to points $P d$ and $P r$ indicated in Fig. 11, whose values are depicted on Table 3, one can check the robustness (in terms of the level of dispersion) of the dynamic responses of the nonlinear damped system. To introduce uncertainties in the optimal and robust solutions, the LHC sampling method is performed, by generating 1,000 samples for each design variable aiming at computing the envelopes of the dynamic responses. The uncertainties introduced in the design variables are the following: $\Delta \rho / \rho=$ $1.5 \%$ for the mass density; $\Delta \mu / \mu=1.5 \%$ for the mass ratio; $\Delta \beta / \beta=1.5 \%$ for the force parameter; and $\Delta \varepsilon_{2} / \varepsilon_{2}=$ $1.5 \%$ for the nonlinear coefficient.

Figure 12(b) shows that the robust optimal solutions are more robust than the deterministic solutions presented in Fig. 12(a). This is demonstrated by the minimal dispersion of the minimal and maximal responses in the neighbourhood of the mean response values. 

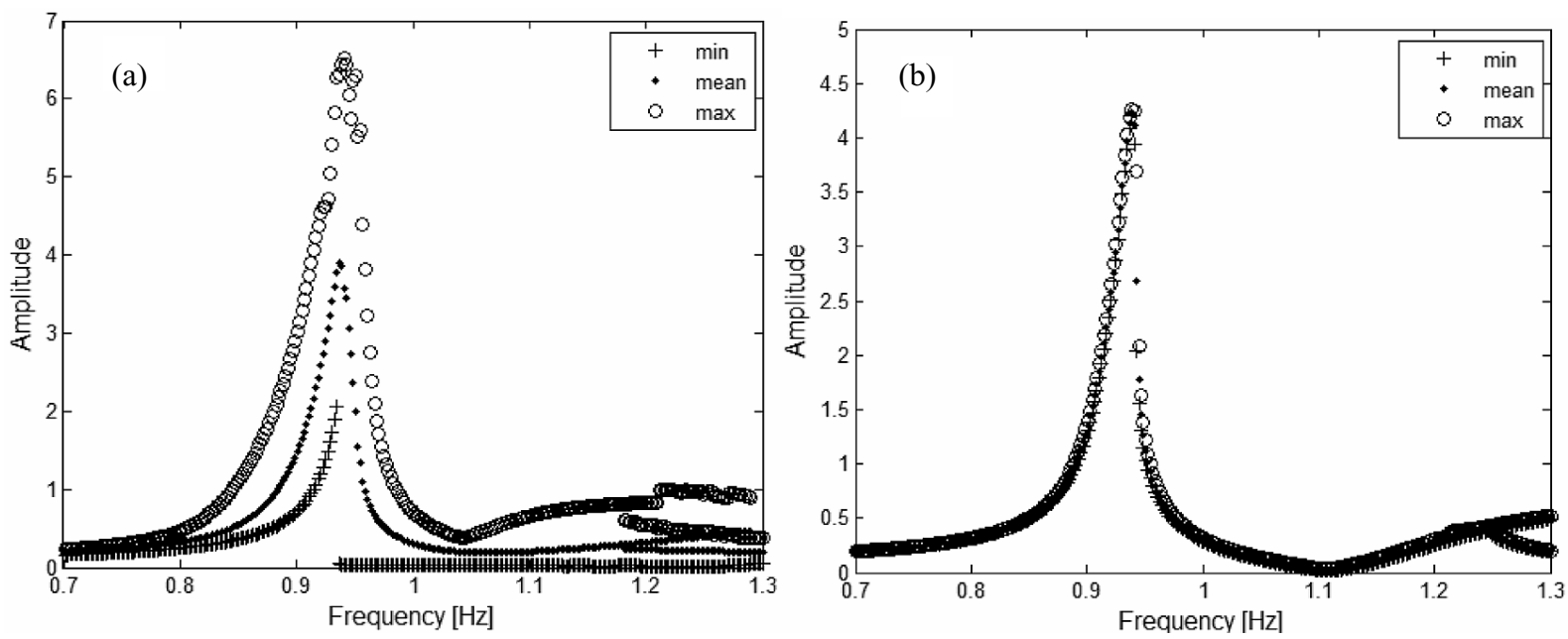

Fig. 12. Envelopes of responses for deterministic (a) and robust (b) optimal solutions.

\section{Concluding remarks}

In this paper the robust optimal design of nonlinear dynamic vibration absorbers was proposed and implemented. The nonlinearities were introduced both in the springs that connect the primary mass to the ground, and the absorber to the primary mass. The originality regarding the multiobjective optimization methodology presented above consists in introducing robustness functions as additional objective functions to be maximized. Each robustness function is associated to an original objective function and is defined to be inversely proportional to the dispersion. Uncertainties on the design variables that characterize the nonlinear dynamic system are introduced directly through a parametric approach, by performing a LHC sampling method.

The numerical applications showed that the sensitivities of dynamic responses convey valuable information about the influence of the design parameters on the dynamic behavior of the nonlinear structure, being also a very useful tool for the design and analysis of modified systems and structural optimization. The choice of the design variables (mass density, mass ratio, force parameter, nonlinearity coefficient) was based on previous knowledge regarding their sensitivities with respect to the amplitude peak and suppression bandwidth. It is worth mentioning that these parameters are directly associated with the effectiveness of the nDVA. However, the limitations of the first-order approximations in the prediction of response variations should not be disregarded.

As demonstrated by the results, the nonlinearity factor is an important parameter to be investigated during the design procedure of nonlinear dynamic systems, due to its contribution to the reduction of the vibration level. However, care must be taken with respect to high values of the nonlinear parameters due to the instabilities that they may introduce in the nonlinear system. This point motivates an important procedure regarding the presented methodology: obtaining the optimal spring nonlinear coefficient that guarantees the best stable solution for a given system.

Finally, the proposed robust optimal design strategy demonstrates the importance of introducing uncertainties in the design variables to be evaluated during the optimization process in order to obtain the optimal and robust design that guarantees the most effective nDVA for a given application.

\section{Acknowledgements}

The authors are deeply grateful to the Brazilian Research Council - CNPq and Minas Gerais State Agency FAPEMIG for the financial support to this research effort (INCT-EIE). The first author is thankful to CNPq for his doctorate scholarship. 


\section{References}

[1] H. Frahm, Device for damping vibrations of bodies, US Patent 989, 958, 1911.

[2] J.P. Den Hartog, Mechanical Vibrations, McGraw-Hill Book Company, Inc., 1934.

[3] B.G. Koronev and L.M. Reznikov, Dynamic Vibration Absorbers: Theory and Technical Applications, John Wiley \& Sons, New York, 1993.

[4] D.A. Rade and V. Steffen, Jr., Optimization of dynamic vibration absorbers over a frequency band, Mechanical Systems and Signal Processing 14(5) (2000), 679-690.

[5] V. Steffen, Jr. and D.A. Rade, Dynamic Vibration Absorber, Encyclopedia of Vibration, Academic Press, ISBN 0-12-227085-1, 2001, 9-26.

[6] L.A. Pipes, Analysis of a nonlinear vibration absorber, Journal of Applied Mechanics 20(3-5) (1953), 515-518.

[7] P.F. Pai and M.J. Schulz, A refined nonlinear vibration absorber, International Journal of Mechanical Sciences 42(3) (1998), 537-560.

[8] H.J. Rice and J.R. McCraith, Practical nonlinear vibration absorber design, Journal of Sound and Vibration 116(3) (1987), 545-559.

[9] C. Zang, M.I. Friswell and J.E. Mottershead, A review of robust optimal design and its application in dynamics, Computers and Structures 83(4-5) (2005), 315-326.

[10] J.R Fonseca, M.I. Friswell and A.W. Lees, Efficient robust design via Monte Carlo sample reweighting, International Journal for Numerical Methods in Engineering 69(11) (2007), 2279-2301.

[11] J. Eschenauer, J. Koski and A. Osyczka, Multicriteria Design Optimization, Springer-Verlag, 1990.

[12] A.M.G. de Lima, M.H. Stoppa, D.A. Rade and V. Steffen, Jr., Sensitivity analysis of viscoelastic systems, Shock and Vibration 13(4) (2006), 545-558.

[13] E. J. Haug, K.K. Choi and V. Komkov, Design Sensitivity Analysis of Structural Systems, Academic Press, 1986.

[14] K.H. Lee and G.J. Park, Robust design for unconstrained optimization problems using the Taguchi method, AIAA Journal 34(5) (1996), $1059-1063$.

[15] K.H. Lee and G.J. Park, Robust optimization considering tolerance of design variables, Journal of Computer and Structures 79(1) (1996), $77-86$.

[16] S. Natsiavas, Steady state oscillations and stability of non-linear dynamic vibration absorbers, Journal of Sound and Vibration 156(2) (1992), 227-245.

[17] A.H. Nayfeh, Perturbation Methods, John Wiley \& Sons, Inc., 2000.

[18] J.C. Nissen, K. Popp and B. Schmalhorst, Optimization of a nonlinear dynamic vibration absorber, Journal of Sound and Vibration 99(1) (1985), 149-154.

[19] J.J. Thomsen, Vibrations and Stability, Springer-Verlag, (2nd Edition), 2003.

[20] S.J. Zhu, Y.F. Zheng and Y.M. Fu, Analysis of non-linear dynamics of a two degree-of-freedom vibration system with non-linear damping and no-linear spring, Journal of Sound and Vibration 271(2) (1992), 15-24.

[21] P. Hagedorn, Nonlinear Oscillations, Clarendon Press, 1988.

[22] http://www.mathworks.com.

[23] A. Florian, An efficient sampling scheme: updates Latin-Hyper-Cube sampling, Probabilistic Engineering Mechanics 7(2) (1992), 123130.

[24] N. Srinivas and K. Deb, Multiobjective using nondominated sorting in genetic algorithms, Technical Report, Department of Mechanical Engineering, Institute of Technology, India, 1993. 

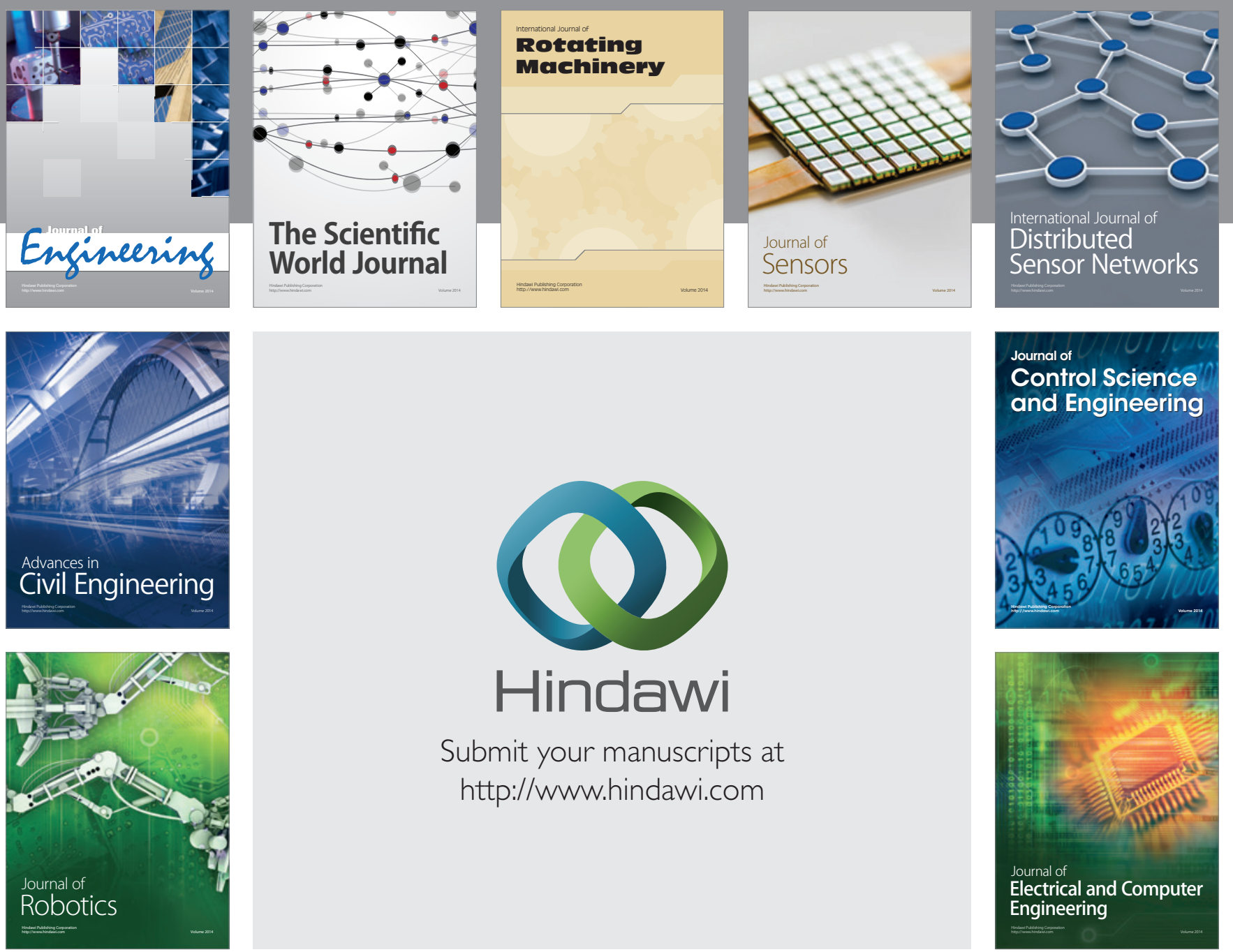

Submit your manuscripts at

http://www.hindawi.com
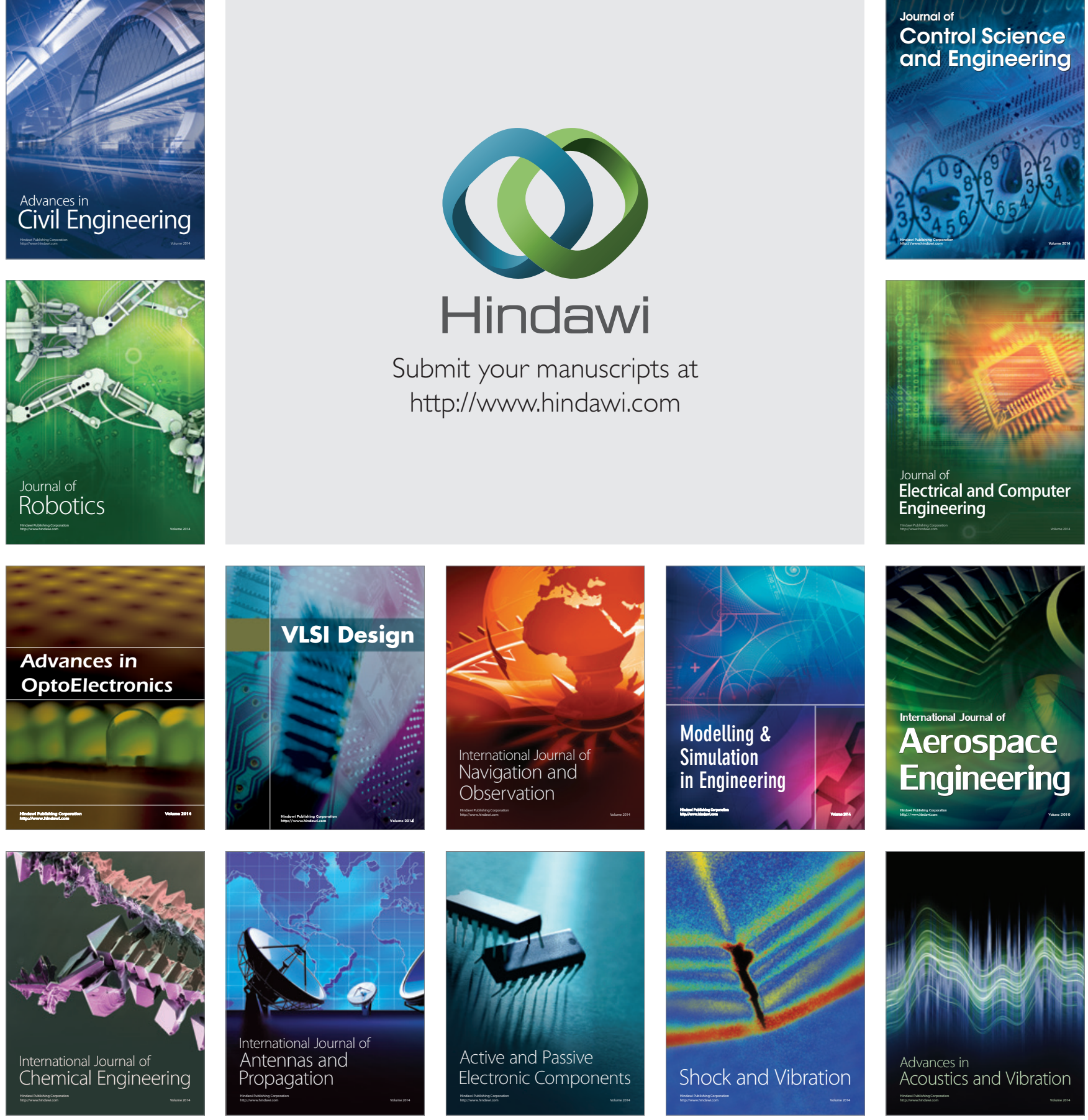